Revue des patrimoines

40 | 2019

Lits historiques. Première anthologie des lits européens du XVe au XIXe siècle

\title{
Le Lit dit de Henri IV du château de Pailhès en Ariège. Histoire, restaurations et perspectives
}

The Bed known as Henri IV's Bed at the Pilhès Castle in the Ariège Department; History, Restorations and Perspectives

\section{Ingrid Leduc}

\section{OpenEdition}

Journals

Édition électronique

URL : http://journals.openedition.org/insitu/24228

DOI : $10.4000 /$ insitu. 24228

ISSN : 1630-7305

\section{Éditeur}

Ministère de la Culture

\section{Référence électronique}

Ingrid Leduc, « Le Lit dit de Henri IV du château de Pailhès en Ariège. Histoire, restaurations et perspectives », In Situ [En ligne], 40 | 2019, mis en ligne le 18 septembre 2019, consulté le 10 décembre 2020. URL : http://journals.openedition.org/insitu/24228 ; DOI : https://doi.org/10.4000/ insitu. 24228

Ce document a été généré automatiquement le 10 décembre 2020.

\section{cc) (†)}

In Situ Revues des patrimoines est mis à disposition selon les termes de la licence Creative Commons Attribution - Pas d'Utilisation Commerciale - Pas de Modification 4.0 International. 


\section{Le Lit dit de Henri IV du château de Pailhès en Ariège. Histoire, restaurations et perspectives}

The Bed known as Henri IV's Bed at the Pilhès Castle in the Ariège Department; History, Restorations and Perspectives

Ingrid Leduc

\section{Henri IV (1553-1610), dernier comte de Foix}

1 Avant de devenir roi de France en 1589, Henri IV fut roi de Navarre et le dernier comte de Foix, à partir de 1572. Bien qu'il n'ait jamais vécu dans son comté, il y séjourna à plusieurs reprises, notamment pour y pacifier les relations entre catholiques et protestants et l'administrer. Force est de constater que ses séjours furent peu nombreux, la présence du souverain n'y étant attestée que sur une période assez courte, entre 1572 et 1587. On sait qu'au printemps 1579, il fut accompagné de Marguerite de Valois, son épouse, et de Catherine de Médicis, sa belle-mère.

2 Ces séjours ont toutefois laissé des traces dans les archives de la chambre des Comptes de $\mathrm{Pau}^{1}$ qui offrent de précieuses informations sur le quotidien du roi de Navarre et de son entourage. On y trouve notamment la mention des dépenses ordinaires du roi, en particulier celles de bouche, les aumônes, cadeaux, ou encore les dépenses dites de «l'Argenterie $»^{2}$.

3 Henri III de Navarre naît en Béarn, à Pau, le 13 décembre 1553. Son père, Antoine de Bourbon (1518-1562), duc de Vendôme, devint roi de Navarre par son mariage avec Jeanne III d'Albret (1528-1572), héritière de la maison de Navarre. En 1566, Jeanne III d'Albret impose le calvinisme comme confession officielle dans le comté de Foix-Béarn.

Dès 1561, Henri III de Navarre fréquente la Cour de France où résidaient le plus souvent ses parents. Pour tenter d'apaiser les tensions entre parti catholique et protestant, Jeanne III d'Albret et Catherine de Médicis décident d'unir leurs enfants, Henri III de 
Navarre et Marguerite de Valois, dont le mariage est célébré le 18 août 1572 à Paris. En 1599, les époux demandent au pape, d'un commun accord, l'annulation de leur mariage et en 1600, Henri IV épouse en secondes noces Marie de Médicis (1573-1642), fille du grand-duc de Toscane. En 1607, le pays de Foix est rattaché à la Couronne de France, tout comme le Béarn et les autres possessions des Bourbon-Navarre.

\section{Le mythe d'un lit royal}

5 Le lit fut conservé jusqu'en 1990 au château de Pailhès, petite commune située au nord de Foix dans la vallée de la Lèze. Il fut mis en vente à cette date par les héritiers du château, ceux-ci devant faire face à de lourdes charges financières (fig. 1).

Figure 1

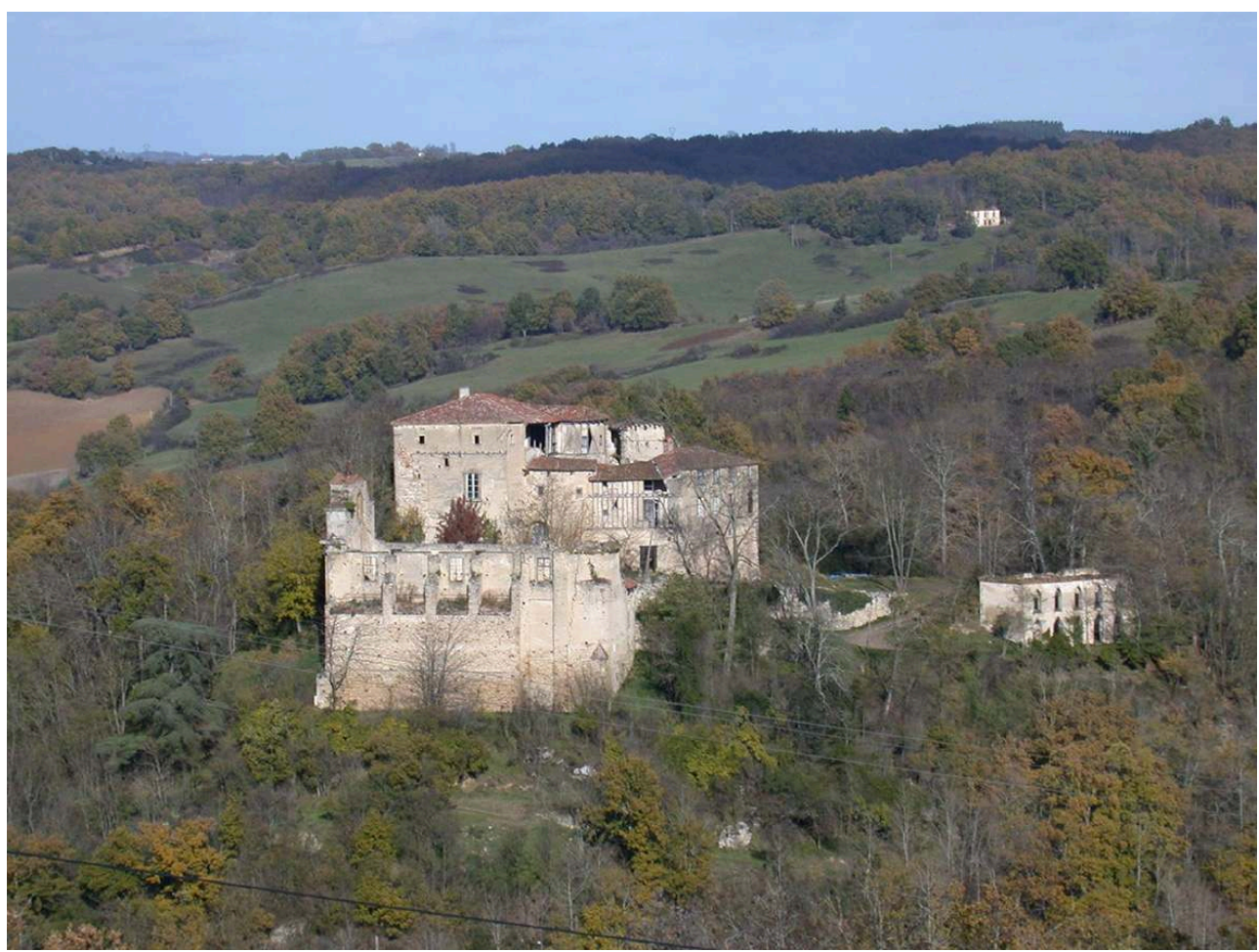

Château de Pailhès (Ariège)

Phot. Thierry Authier. (c) Conseil départemental de l'Ariège.

Situé sur un piton rocheux, au carrefour des importantes voies de circulation entre Toulouse et l'Andorre, le château fut occupé à partir du $\mathrm{XIII}^{\mathrm{e}}$ siècle par la puissante famille des Villemur-Pailhès qui le posséda jusqu'au début du XVIII siècle. C'est sous le règne d'Henri III de Navarre que Pailhès connut son heure de gloire. Le souverain confia en effet à Jacques de Villemur, baron de Pailhès, de nombreuses charges. Le bourg de Pailhès devint ainsi le centre de la vie militaire et administrative du comté. Le prestige de la seigneurie fut renforcé par les relations amicales qu'entretint Henri III de Navarre avec Jacques de Villemur et son fils Blaise, comme en témoigne la correspondance conservée aux archives départementales de l'Ariège et dans plusieurs collections privées ${ }^{3}$. 
7 Nommé gouverneur du comté de Foix en 1566, Jacques de Villemur occupait une fonction majeure qui le hissait au premier rang des seigneurs ariégeois. Dès la fin du Moyen Âge, les souverains du Béarn étaient représentés dans le comté de Foix par deux officiers. Le sénéchal exerçait la justice; les affaires militaires étaient entre les mains d'un gouverneur désigné par les souverains de Navarre. L'aménagement d'une chambre pour le roi au château de Pailhès n'avait donc rien donc de surprenant. Elle témoigne avant tout de la volonté des seigneurs de Pailhès de mettre en avant la relation privilégiée qu'ils entretiennent avec les souverains de Navarre, a fortiori lorsque celuici devint roi de France.

8 À ce jour, aucun document connu n'atteste de la commande ou de l'installation du lit (fig. 2). Il est toutefois possible qu'Henri de Navarre ait séjourné au château de Pailhès du 30 novembre au $1^{\text {er }}$ décembre 1579 , à l'occasion de son voyage de retour vers le château de Mazères, où il réside le plus souvent lors de ses déplacements dans le comté. Une tradition orale voudrait que des dames de la noblesse locale aient brodé les rideaux au point de Hongrie du lit, ce que l'étude technique dément, du fait de de la complexité de ces garnitures.

Figure 2

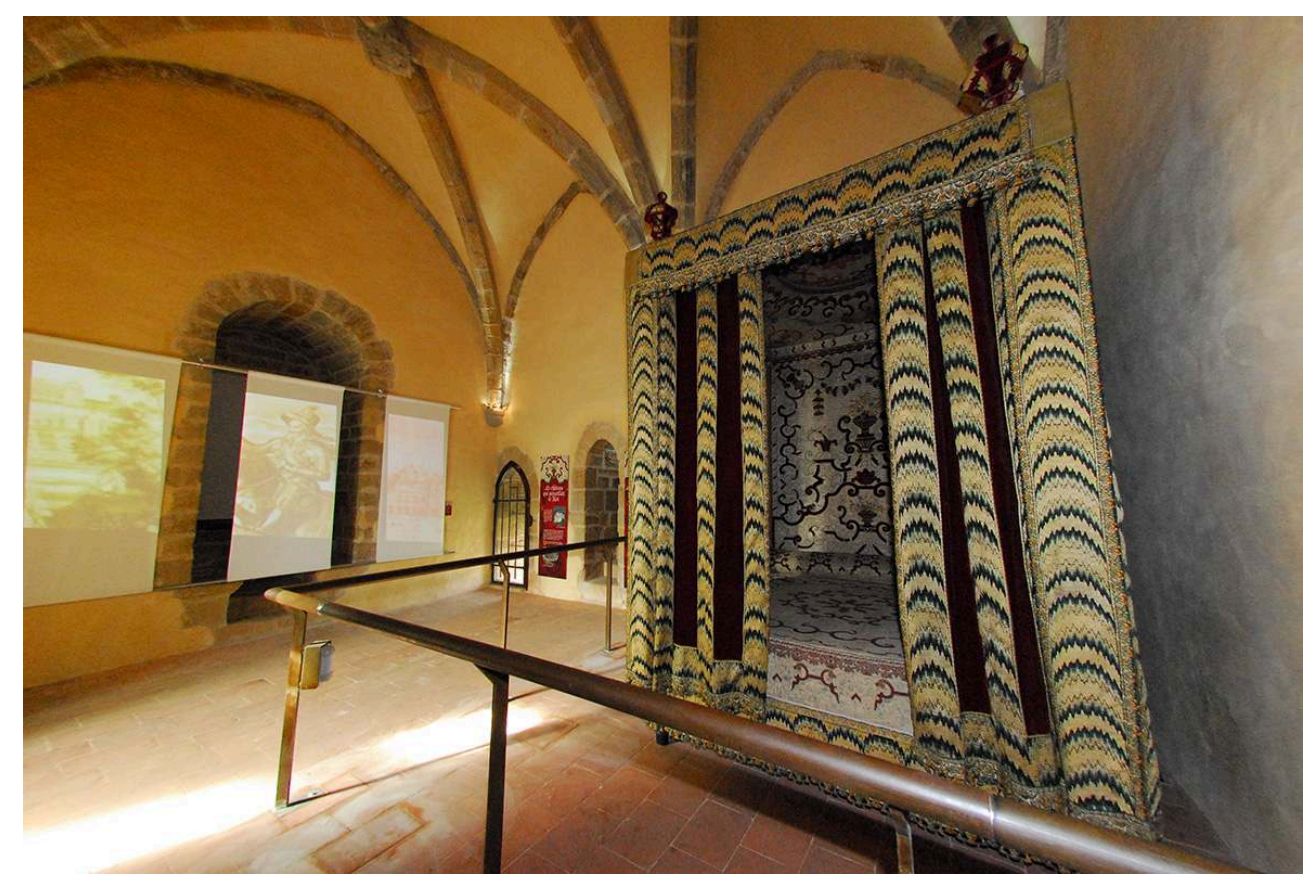

Lit, château de Pailhès (Ariège).

Phot. Thierry Authier. (c) Conseil départemental de l'Ariège.

9 Le lit demeura dans la même pièce, au premier étage du château de Pailhès, pendant près de quatre siècles. Connue depuis le XVII ${ }^{e}$ siècle sous le nom de "chambre du roi ", cette pièce était attenante à une grande salle ${ }^{4}$.

Le lit, classé au titre des monuments historiques en $1965^{5}$, fut acquis en 1991 par le conseil général de l'Ariège pour son musée départemental. Présenté quelques années au palais des Évêques de Saint-Lizier, il rejoint en 2006 le château de Foix dans la tour dite du Milieu, où se situe une chambre d'apparat du XIV ${ }^{e}$ siècle. Le châlit et les garnitures textiles furent restaurés de 1994 à 1999 par l'atelier d'Isabelle Bédat 
(Vanves) pour les textiles et l'entreprise Férignac (Hautefort, Dordogne), puis la société Malbrel (Capdenac, Lot) pour les bois. À cette occasion, il fut démonté, ce qui permit de mieux comprendre sa mise en œuvre. Le lit ne présentait pas de traces de moisissure mais une importante infestation, notamment au niveau de la laine du matelassage, qui avait entraîné de nombreuses dégradations et des lacunes, en particulier sur la courtepointe. Les interventions de restauration sur les textiles ont été réalisées entre 1998 et 1999. Le parti retenu fut de préserver en l'état les tissus, qui au cours du temps, ont été peu réparés ou modifiés.

11 Les garnitures ont été nettoyées par micro-aspiration sur l'envers et l'endroit. Tous les fils détériorés ont été refixés, sans ajout de broderie. Les zones trop fragilisées ont été recouvertes de crêpeline de soie teintée en rouge au niveau des applications de velours de soie cramoisi pour donner une certaine unité visuelle à l'ensemble.

\section{Un meuble composite}

12 Le lit tel que nous le connaissons aujourd'hui est le résultat d'un réassemblage, tant au niveau du châlit que des garnitures textiles, bien que la plupart des composantes semblent bien dater du XviI ${ }^{e}$ siècle. Il est ainsi difficile de faire entrer ce lit dans une typologie précise car la garniture extérieure a été conçue pour un lit à colonnes tandis que la garniture intérieure est celle d'un lit «à la française ». Il se présente à ce jour sous la forme d'un lit de bout, dit "à la française ${ }^{6}$ ", composé d'un châlit en bois avec sommier, quatre quenouilles en hêtre anciennes, un ciel ancien, une ceinture haute ancienne en frêne et une ceinture basse ancienne en frêne qui rigidifient le lit.

Les quatre pieds, en forme de colonnes tournées, sont assemblés avec la ceinture dans leur partie basse carrée au moyen de tenons et mortaises et vis de rappel. Sous le lit, des goberges ${ }^{7}$ assemblées à queue d'aronde supportent le récent sommier.

La couche est constituée d'un cadre de lit avec un dossier de tête ancien et d'un sommier aujourd'hui restitué.

Plusieurs goberges qui formaient un sommier creux ont été retirées lors de la dernière restauration et sont aujourd'hui conservées dans les réserves du musée départemental. Les matelas et éléments textiles de la couche n'ont pas été conservés.

Dans les années 1990, le coucher a été restitué au moyen d'une caisse de bois lattée en peuplier et gainée de molleton. Le panneau de latté est constitué d'une multitude de baguettes de bois collées entre elles et recouvertes par un placage. Le latté fut considéré comme une bonne alternative pour sa compatibilité avec les textiles historiques car c'est un panneau qui contient une faible concentration de colle. Le bois de peuplier, dont le niveau d'acidité est peu élevé, concourt à la préservation des fibres textiles. L'aspect général demeurait toutefois peu satisfaisant car trop raide.

17 En partie haute, des tiges de métal forgé sont fichées sur les quenouilles et permettent d'enfiler d'abord l'ancienne ceinture haute du ciel (restaurée mais en mauvais état) puis un nouveau cadre en noyer servant de support aux rideaux et aux pentes. Des équerres forgées sont clouées sur le cadre et supportent des tringles modernes sur trois côtés du lit. Ces tringles servent à suspendre les courtines et les rideaux.

La tête de lit est découpée, en partie supérieure, en forme d'arabesques. Le dossier est un rideau rectangulaire. Le centre de ce dossier est orné d'une imposante broderie de vases à fleurs réalisés en peinture à l'aiguille (point passé plat empiétant). Le vase 
central est ourlé de faisceaux de fils noirs maintenus par des ponts réguliers (point de couchure).

Figure 3

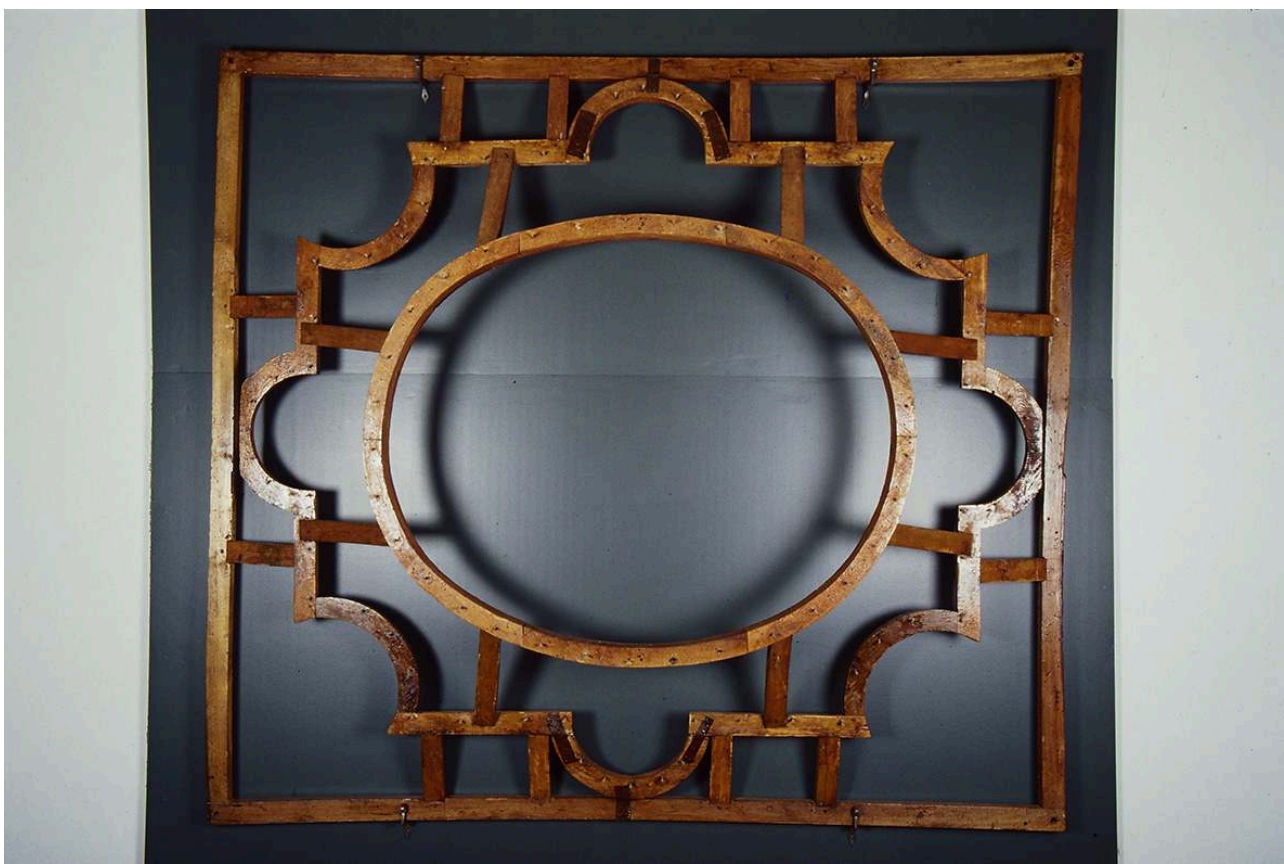

Dais du lit, château de Pailhès (Ariège).

Phot. Thierry Authier. (c) Conseil départemental de l'Ariège.

Le lit est couvert par un dais particulièrement remarquable (fig. 3). De forme rectangulaire, il se déploie ensuite en hauteur pour former dôme en son centre. La jonction se fait au moyen de tasseaux de bois assemblés de manière à former un décor d'arabesque. Cette structure est façonnée dans un bois peu tannique comme le hêtre ou le frêne et assemblée à tenon et mortaise et parfois cloutée, sans qu'il nous ait été possible jusqu'ici d'en observer complètement l'assemblage. Le ciel de lit est composé de trois niveaux de tissus en coupole : un petit fonds ovale et un second élément textile en forme et en volume pour les deux niveaux inférieurs. La petite pente est cousue le long des bords du ciel de lit. Les tissus sont tendus à l'aide de pitons plantés dans le bois $\mathrm{du}$ ciel qui viennent s'enfiler dans un galon troué autour du rond du médaillon. Les quatre coins sont maintenus en tension à l'aide de pitons et d'anneaux. Lors de la dernière restauration, la partie haute a été couverte d'un Tyvek ${ }^{\oplus}$ (non tissé en fibres de polyéthylène) de protection. Le ciel de lit est surmonté à l'avant de deux pommes en bois peintes et partiellement recouvertes de velours rouge.

Les dimensions actuelles du lit (longueur : $226 \mathrm{~cm}$, largeur : $195 \mathrm{~cm}$, hauteur : $312 \mathrm{~cm}$ ) résultent des deux campagnes de restauration. Les quenouilles ont ainsi été rallongées à deux reprises, une première fois, à une date inconnue, de $18,5 \mathrm{~cm}$ au moyen d'un enfourchement chevillé. En 1999, l'entreprise Férignac a procédé à une seconde augmentation par entage chevillé en forme de $\mathrm{V}$ de $16,5 \mathrm{~cm}$. Le lit apparaît ainsi trop haut par rapport à la hauteur des rideaux qui s'arrêtent désormais au niveau de la couche. 
Les éléments anciens du châlit présentent de nombreuses dégradations, notamment des attaques d'insectes xylophages qui compromettent par endroits la cohésion du bois.

L'ensemble témoigne d'une grande maîtrise de la menuiserie, tant dans le façonnage à la gouge de ces quenouilles que dans l'assemblage des bois du ciel.

Les garnitures de textiles du lit sont particulièrement remarquables et relativement bien conservées. Une fois le lit monté, elles recouvrent l'ensemble du châlit et du dais. L'observation des différentes composantes permet de comprendre que ce sont deux garnitures, l'une datant du début du XVII ${ }^{\mathrm{e}}$ siècle et l'autre de la seconde moitié du XVII siècle, qui ont été remontées ensemble à une époque indéterminée pour former ce meuble.

24 À l'extérieur, la garniture du lit consiste en broderies au point de Hongrie (fig. 4). La broderie au point de Hongrie est une technique ancienne déjà attestée au $\mathrm{XVI}^{\mathrm{e}}$ siècle qui utilise un procédé de mise en œuvre des fils par séries, créant un motif de zigzag et un effet de dégradé des couleurs. Ce procédé était souvent utilisé pour les garnitures de lit et les tentures murales en raison de son aspect couvrant et de sa relative rapidité d'exécution.

Figure 4

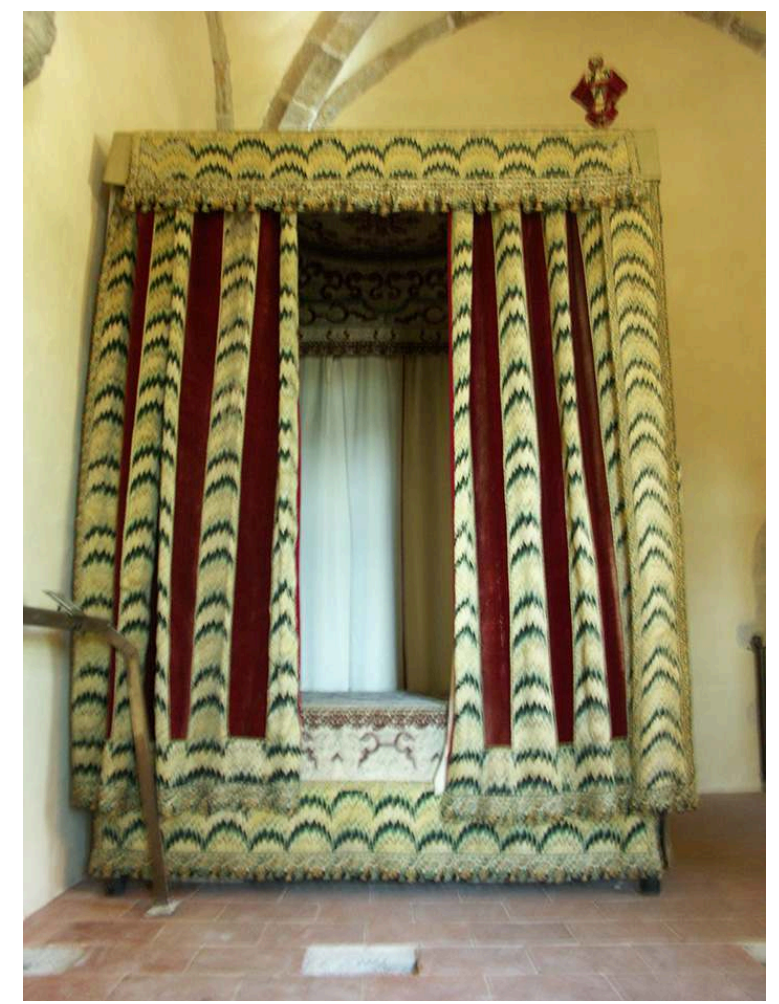

Garniture extérieure au point de Hongrie, château de Pailhès (Ariège).

Phot. Thierry Authier. (c) Conseil départemental de l'Ariège.

La broderie du lit de Pailhès présente ici un motif de flamme pour la gamme des fils de laine vert moyen, vert très foncé, vert sapin tandis qu'un motif de chevrons est utilisé pour les fils de soie jaune, vert clair et noir, accrochant ainsi la lumière de façon subtile. Le rapport de restauration établi en 1998 par Isabelle Bédat, restauratrice de textiles anciens, permet de mieux comprendre la mise en œuvre de cette broderie. La toile de 
trame est aérée, en fibres cellulosiques beige. Le nombre de fils de chaîne est de sept fils simples. Très souple, cette toile permet un passage aisé des fils de broderie souvent épais comme la laine. Elle est identique sur l'ensemble des éléments. La broderie est quant à elle réalisée au moyen de onze fils de laine (vert et orangé) et de soie (jaune, vert, noir). Le rapport de dessin de compose de huit ondes. Le faible nombre de fils de broderies, astucieusement alternés, et le jeu de chevrons monochromes de fils de soie jaune animent la surface de la broderie et accrochent la lumière de manière subtile.

Les pentes hautes et basses cachent le dais et le tour du lit. Les six rideaux, également brodés au point de Hongrie, sont associés à des bandes de velours de soie rouge. Ils sont actuellement doublés par un satin de soie beige de restauration qui vient en remplacement de la doublure de soie ancienne en mauvais état. La toile de renfort a toutefois été conservée, l'ensemble étant maintenu par des points de bagage. Les panneaux sont terminés sur leurs trois côtés par des bandes de broderies à motifs de fleurs aux bords découpés de manière à individualiser chaque fleur. Les quatre rideaux des côtés ont subi l'adjonction récente d'une bande de drap de laine rouge, probablement pour remplacer la bande d'origine détériorée. Cette association de textiles (soie, laine, velours) peut laisser penser qu'il s'agit là d'un meuble d'hiver.

Plusieurs autres lits peuvent être mis en relation avec la garniture au point de Hongrie du lit de Pailhès, en particulier le lit de la chambre Charles IX au château de Talcy (Loiret-Cher) installé depuis le xVIII ${ }^{e}$ siècle ${ }^{8}$. Le meuble était composé d'une tenture murale flottante et de sièges garnis du même textile. La tenture flottante a toutefois disparu. En effet, au milieu du XvII ${ }^{e}$ siècle, la tendance est de coordonner pour le décor intérieur tapissé tentures murales et meubles?.

La mise en œuvre du lit de Talcy est assez proche de celui de Pailhès car on retrouve le motif de flammes dans des dégradés de couleurs en partant du jaune jusqu'au bleu et au vert et en passant par l'ocre et le brun. Les rideaux au point de Hongrie sont associés à des bandes de velours de soie marron et terminés par un galon de broderie. On trouve également au château de Montal (Lot), dans la chambre aménagée pour Mme Fenaille ${ }^{10}$, un lit ayant conservé ses pentes et sa courtepointe du XvII ${ }^{\mathrm{e}}$ siècle ainsi que plusieurs sièges et une tenture flottante au point de Hongrie. Enfin, il convient de mentionner le lit de la Grande Chambre de Parham Hall ${ }^{11}$ (Suffolk), en Angleterre, dont les pentes hautes et basses ainsi que les rideaux présentent également de fortes similitudes avec les lits de Pailhès et de Talcy.

L'observation détaillée du lit de Pailhès permet de comprendre que la garniture au point de Hongrie a été adaptée au châlit actuel qui ne correspond pas aux dimensions originelles du lit. Au niveau des pentes hautes, on peut ainsi observer, dans les angles, des rajouts modernes de textile brun qui permettent de recouvrir la ceinture haute. D'autre part, on remarque la présence sur les rideaux de pied d'un ruban métallique qui marque les angles et unit par deux les rideaux. Il s'agit probablement là d'un ruban datant du XIX ${ }^{e}$ siècle. 


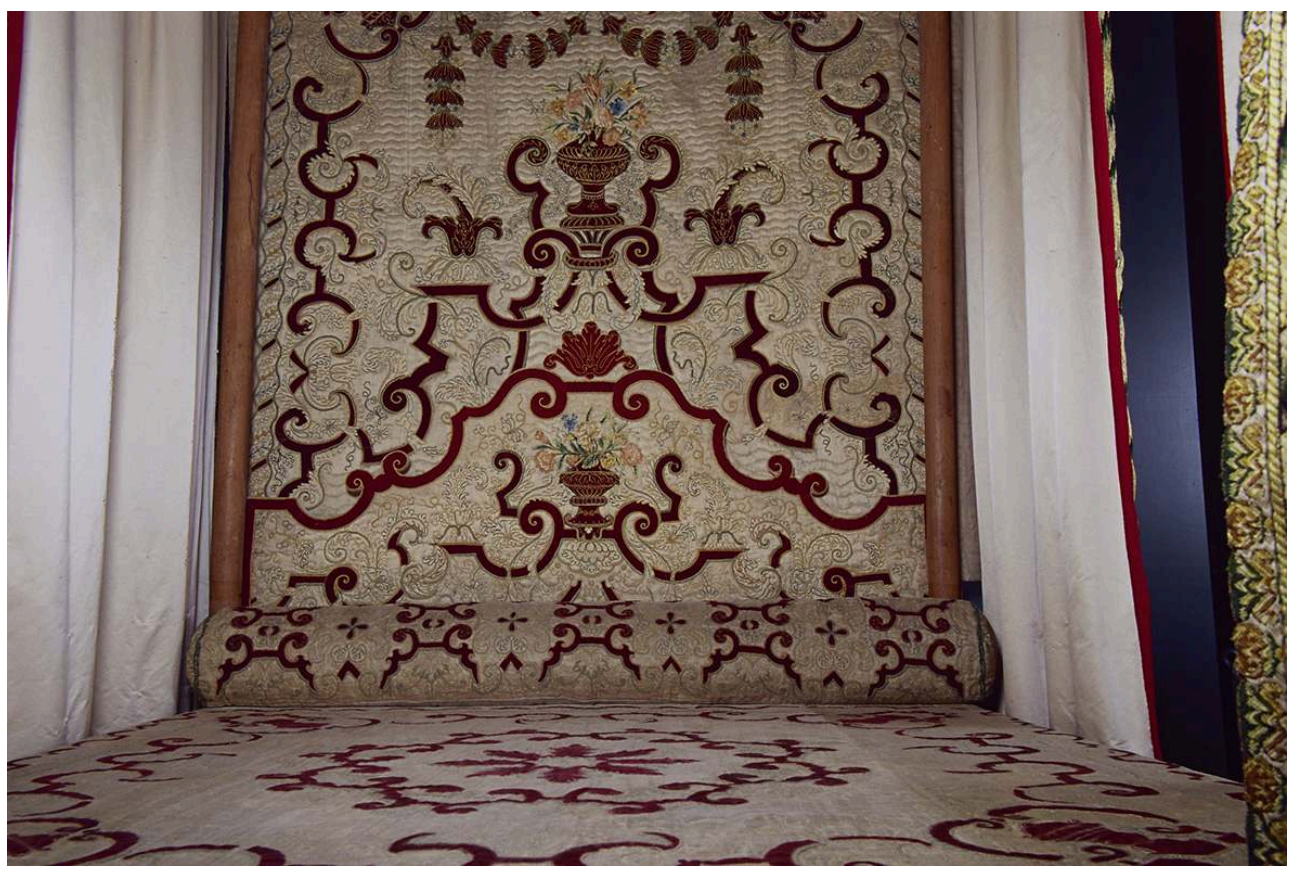

Garniture intérieure en satin de soie, château de Pailhès (Ariège).

Phot. Thierry Authier. (c) Conseil départemental de l'Ariège.

La garniture intérieure du lit (courtepointe, dosseret, fond de lit et ciel de lit) est en satin de soie beige matelassé de vagues (fig. 5). Cette garniture textile est homogène et les différents éléments sont réalisés avec les mêmes techniques. Il s'agit d'un assemblage de plusieurs couches de tissus: une toile en fibres végétales de couleur naturelle, sur laquelle repose un rembourrage (identifié comme une bourre de laine par Isabelle Bedat, restauratrice de textiles) recouvert d'un satin de soie crème. La soie est brodée au moyen de fils de soie colorés et des décors d'applications, à motifs d'arabesques, sont réalisés avec un ruban de velours rouge (soie ?) ourlés de lézardes, de marabouts en passementerie et de ganses vertes et jaunes. Cet ensemble peut être daté de la seconde moitié du $\mathrm{xVII}^{\mathrm{e}}$ siècle. Ses dimensions sont également plus importantes. Pour assembler (à une date inconnue) les deux meubles, on a dû replier une partie de la courtepointe et probablement augmenter la hauteur des quenouilles. Cette garniture présente une mise en œuvre d'une grande qualité. Le dessous est réalisé en fibre cellulosique, puis vient un matelassage de bourre de laine ancienne recouvert par un satin de soie de couleur beige agrémenté par un savant motif de coutures en vagues. Ces étoffes matelassées étaient relativement fréquentes au XVII ${ }^{\mathrm{e}}$ siècle pour les garnitures intérieures des lits, comme en témoignent notamment les inventaires du château de Grignan qu'avait pu consulter Xavier Bonnet, tapissier et historien de l'art.

Sur le satin de soie beige, des broderies et des applications de passementerie et de velours de soie cramoisi constituent un décor d'arabesques somptueux et chatoyant déployé autour de vases de fleurs peints à l'aiguille. Les motifs de velours de couleur pourpre constituent un décor géométrique assez proche des arabesques de Jean Bérain et de la marqueterie Boulle et encadrent des tulipes, narcisses, œillets et roses brodés de fils de soie colorés. Ce motif de fleurs au naturel peintes à l'aiguille de couleurs vives 
et pastel est caractéristique du tissu d'ameublement en France de la fin du XVII et du début du XVIII ${ }^{e}$ siècle (fig. 6).

Figure 6

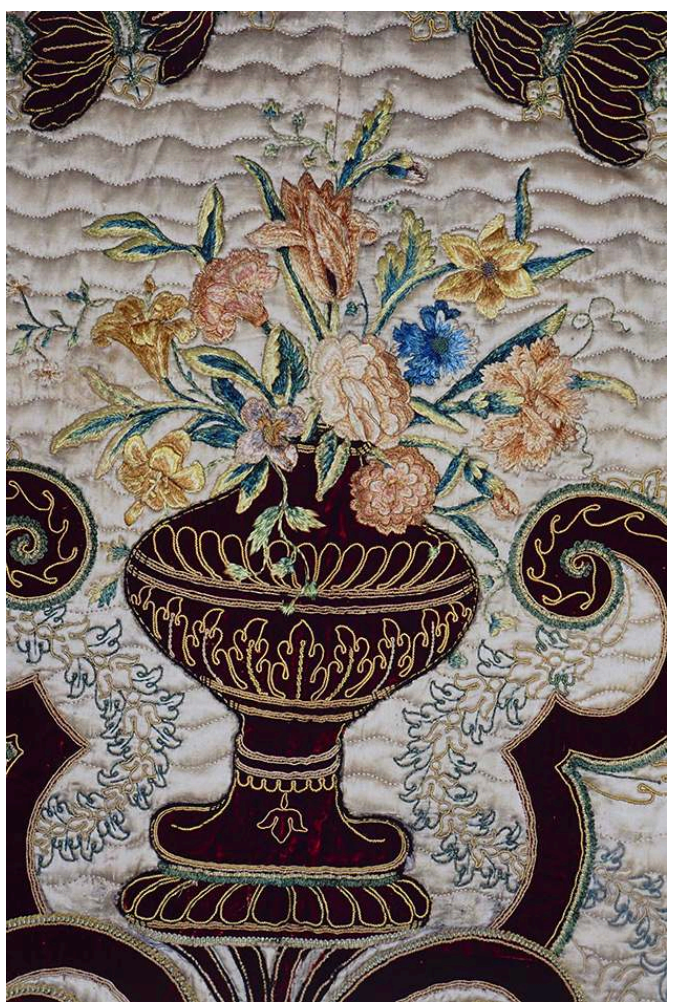

Motifs de fleurs au naturel et vase peints à l'aiguille, château de Pailhès (Ariège).

Phot. Thierry Authier. (c) Conseil départemental de l'Ariège.

L'ornement floral tient une place particulière dans la broderie du xvII siècle, en particulier dans deux catégories : l'ameublement (et en particulier le décor des lits) et la broderie liturgique ${ }^{12}$.

La fidèle représentation des fleurs rappelle l'intérêt du XvII ${ }^{\mathrm{e}}$ siècle pour les nouvelles variétés et le développement de la botanique. Le botaniste Jean Robin (1550-1629) publie en 1601 avec le soutien du roi un catalogue des variétés de fleurs indigènes et exotiques cultivées à Paris ${ }^{13}$. En 1633 est créé à Paris le Jardin des plantes.

Les fleurs sont présentes dans les bordures décoratives mais elles constituent aussi souvent le motif principal du décor. Ce sont pour la plupart des fleurs nouvellement connues en Europe et importées du Proche-Orient, d'Asie et d'Amérique qui suscitent un intense engouement et se monnayent à prix d'or.

Parmi les fleurs les plus représentées figurent les roses, les lys ou les ancolies traditionnels mais surtout les variétés nouvellement introduites comme la tulipe, les fritillaires, les anémones, les œillets, les narcisses, les jacinthes, et toutes les nouvelles variétés d'iris, de lys et de primevères. La disposition des fleurs dans le décor varie : on les trouve encore fréquemment dans les bordures mais aussi isolées ou regroupées en bouquets ou encore disposées dans des vases ouvragés. Il ne reste malheureusement qu'un très petit nombre de lits ou de garnitures conservés, dont le lit de Pailhès. 

diffusion des modèles. Le souhait de conserver le souvenir de ces fleurs précieuses et de faire connaître les nouvelles variétés donne lieu à la production de recueils de plantes gravées, Les Florilèges. L'initiateur de ce genre d'ouvrages en France fut Pierre Vallet, brodeur ordinaire du roi, qui publia en 1608 son fameux Jardin du Roy très chrestien Henry IV, roy de France et de Navarre et dédié à la Royne, dont les planches gravées illustraient les plantes du jardin de Jean Robin ${ }^{14}$.

Le lit de Pailhès, exceptionnel, semble ainsi avoir été fabriqué par des artisans particulièrement qualifiés. Sans qu'il soit possible de caractériser une provenance, on peut rappeler la présence d'ateliers de brodeurs à Toulouse, en particulier aux XVI et $\mathrm{XVII}^{\mathrm{e}}$ siècles. La richesse inégalée de Toulouse et du Lauragais au Xvi $\mathrm{e}^{\mathrm{e}}$ siècle, grâce à la culture du pastel, favorise le développement d'un artisanat de luxe. Le comté de Foix (actuel département de l'Ariège) connut également un foyer artistique au $\mathrm{xvI}^{\mathrm{e}}$ siècle autour de Mirepoix, grâce au mécénat de la famille de Lévis-Mirepoix, qui faisait partie de la maison comtale.

\section{Un nouvel écrin dans l'ancien palais des Gouverneurs}

En 2015, le conseil départemental de l'Ariège a lancé un grand projet de restauration du château de Foix et de l'ancien palais des Gouverneurs, situé à proximité immédiate. Le château, bien connu, est le monument le plus visité du département avec une fréquentation annuelle d'environ 90000 visiteurs. Il abrite depuis 1951 une partie des collections du musée départemental. Le projet prévoit la restauration des salles historiques du château et la création d'un nouvel espace muséal. Les conditions d'accès et de sécurité seront également améliorées. Le conseil départemental s'est en effet engagé dans une politique patrimoniale ambitieuse en accompagnant le développement de ses sites remarquables et en finançant largement la restauration de son patrimoine. Dans le cadre de ce projet, les collections du musée départemental ont été restaurées et installées dans les nouveaux espaces muséographiques du palais des Gouverneurs, de manière à offrir au visiteur un parcours de visite confortable et de bonnes conditions de conservation pour les collections.

Depuis toujours, ce lit a donné lieu à de nombreuses légendes, bien que la présence du souverain dans le comté de Foix ne soit attestée qu'à cinq reprises. Un dépouillement exhaustif des archives financières conservées aux archives départementales des Pyrénées-Atlantiques a permis de bien documenter les séjours qu'il fit entre juin 1572 et août 1589. Les cahiers de la série B 43 mentionnent notamment les dépenses réalisées entre fin novembre et début décembre 1579. Bien que le séjour à Pailhès ne soit pas précisément indiqué, l'hypothèse d'un séjour au château et du repos du roi dans le lit exceptionnel s'est rapidement imposée et a nourri de nombreux récits populaires, contribuant à alimenter le mythe jusqu'à nos jours. Il a donc été décidé de présenter le dans une period room permettant d'évoquer une chambre d'apparat méridionale du $\mathrm{XVII}^{\mathrm{e}}$ siècle. Le projet d'aménagement de cette salle répond à deux ambitions, offrir des conditions de conservation optimales, notamment des textiles anciens, mais également permettre au public de découvrir cet élément emblématique du patrimoine ariégeois. Pour le lit, on a choisi de remplacer le sommier moderne actuel par des matelas réalisés par Amandine Cambet, tapissière historique. 
40 Autour du lit seront également présentés des objets, œuvres d'art et pièces de mobilier du XVII ${ }^{e}$ siècle qui étaient en usage dans la France méridionale. On a ainsi privilégié une sélection d'œuvres régionales, plus particulièrement conservées en Ariège, notamment des pièces exceptionnelles datées du XVII ${ }^{e}$ siècle et protégées au titre des monuments historiques, un buffet haut en noyer ciré, une chaise à bras, un miroir, deux chandeliers en bronze gravé, un coffret en argent repoussé, une copie ancienne de la Vierge de Lorette d'après Raphaël, un tableau attribué à Alonso Cano représentant l'Apparition de la Vierge à saint Antoine. Un décor sobre accompagnera les collections, notamment une toile tendue en velours rouge placée contre le mur à l'arrière du lit permettant de diffuser de manière douce le flux de la climatisation. Des tomettes seront posées au sol, sur le modèle de la chambre du roi du château de Pailhès, un rideau et une tringle seront recréés par Michel Chauveau et Amandine Cambet, tapissiers historiques. Les murs seront laissés dans une couleur claire pour les parties modernes et en pierre apparente pour les murs anciens du bâtiment. Une mise à distance permettra de circuler uniquement sur un côté de la salle, face au lit. Le climat sera contrôlé et régulé automatiquement. Le projet d'éclairage, confié à Jean-Jacques Ezrati ${ }^{15}$, devra à la fois être satisfaisant pour le visiteur et garantir la conservation des textiles. Pour cela, M. Ezrati a conçu un éclairage dynamique qui suit un scénario de mise en lumière du lit d'environ deux minutes. L'éclairage d'ambiance sera réalisé à partir de deux projecteurs à focale variable afin d'obtenir un éclairage diffus et harmonieux de toute la pièce.

41 L'éclairage du lit sera réalisé à partir de projecteurs à focalisation, au moins un projecteur sera placé en contre-plongée de manière à éclairer l'intérieur du lit. Tous les projecteurs seront individualisés et connectés à des rails DALI de manière à réaliser le scénario le mieux adapté à la présentation du lit. De ce fait, les textiles ne sont jamais éclairés de manière continue mais successivement, élément par élément. Le dispositif d'éclairage est déclenché par un capteur de présence et la period room est maintenue dans la pénombre en l'absence de visiteur.

Préalablement à ce déménagement, une étude du lit a été confiée à Émilie Enard et Nadège François, restauratrices de textiles, et à Christian Schmitters, restaurateur en ébénisterie, de manière à préparer son démontage, établir un constat d'état détaillé et anticiper les restaurations nécessaires. La courtepointe, en particulier, nécessite des interventions importantes et un remplacement de la crêpeline posée lors de la dernière restauration. Des reprises sont à prévoir sur les rideaux au point de Hongrie. Cette étude sera également l'occasion d'envisager une reprise des restaurations actuelles des quenouilles, de manière à rétablir une plus grande harmonie entre le châlit et les ensembles textiles.

\section{NOTES}

1. - Conservées aux archives départementales des Pyrénées-Atlantiques. 
2. - PÉBAY-CLOTTES, Isabelle. «Les archives financières, source de l'histoire des séjours d'Henri de Navarre dans le comté de Foix ». Archives ariégeoises, 2014, nº 6, p. 101-161.

3. - LA HITTE, Charles de, vicomte. « Lettres inédites de Henry IV à M. de Pailhès, gouverneur du comté de Foix, et aux consuls de la ville de Foix, 1576 - 1602 ». Mémoires de la Société historique de Gascogne, 1886.

4. - BOUSCATEL, Vincent. Pailhès. Un Château, une terre, des seigneurs. Pailhès : Vincent Bouscatel, 2010 , p. 45.

5. - Voir le site : https://www.pop.culture.gouv.fr/notice/palissy/PM09000521.

6. - Lit fait pour être perpendiculaire au mur et adossé à celui-ci par le chevet de tête.

7. - Petites traverses de bois placées sur le fond d'un lit et qui servent à supporter le matelas (TLF).

8. - Voir le site : http://www.chateau-talcy.fr/ [consulté le 16/07/2019].

9. - KRAATZ, Anne. « Le tissu d'ameublement sujet orné et objet d'ornement. Usages en France et influences italiennes au XVII siècle". Dans COQUERY, Emmanuel (dir.). Rinceaux et figures. L'ornement en France au XVII siècle. Saint-Rémy-en-l'Eau/Paris : Monelle Hayot/musée du Louvre, 2005, p. 125-139, ici p. 136.

10. - Maurice Fenaille, industriel, grand collectionneur d'art ancien, mécène et auteur, entre autres, d'ouvrages sur l'histoire de la tapisserie, fit l'acquisition du château de Montal en 1908.

11. - Voir le site: https://www.parhaminsussex.co.uk/the-house/what-to-see/the-greatchamber/ [consulté le 16/07/2019]. Il indique, apparemment sans fondement, et de façon quelque peu fantaisiste, que les tapisseries du dais, du dosseret et de la courtepointe datent de 1585 et furent " probably commissioned by Marie de Medici [en fait Catherine], sister-in-law [en fait belle-mère] to Mary Queen of Scots». Les rideaux, tentures et cantonnières dateraient des années 1620 .

12. - VÉRON-DENISE, Danièle. «La place et le rôle de la fleur dans la broderie du XVII ${ }^{\mathrm{e}}$ siècle ». Dans COQUERY, Emmanuel (dir.). Op. cit., p. 101-112, ici p. 101.

13. - ROBIN, Jean. Catalogus stirpium tam indigenraum quam exoticarum quae Lutetiae coluntur. Paris : Philippe du Pré, 1601.

14. - VÉRON-DENISE, Danièle. Art. cit., p. 106.

15. - Spécialiste des éclairages muséographiques, il est l'auteur, entre autres, d'un récent ouvrage sur l'éclairage d'exposition (Eyrolles, 2014).

\section{RÉSUMÉS}

Avant d'être roi de France, Henri IV fut roi de Navarre, sous le nom de Henri III, et comte de Foix. Bien qu'il n'ait jamais vécu dans son comté, il y séjourna à plusieurs reprises entre 1572 et 1587. C'est au château de Pailhès, situé au nord de Foix, que fut conservé jusqu'en 1990 un lit remarquable $\mathrm{du} \mathrm{xVII}^{\mathrm{e}}$ siècle. Le souverain entretint des relations privilégiées avec Jacques de Villemur-Pailhès et le nomma gouverneur du comté en 1566. L'aménagement d'une chambre du roi au sein du château n'avait donc rien donc de surprenant et témoigne du prestige de cette seigneurie. À ce jour, aucun document connu n'atteste de la date de commande ou d'installation du lit. Conservé aujourd'hui au château de Foix, il a subi une importante restauration entre 1994 et 1998 tant au niveau du châlit que de ses garnitures textiles. L'étude de ce lit montre qu'il s'agit d'un meuble composite. Le châlit en bois a été réadapté à une époque inconnue, puis lors de sa 
restauration, de manière à adapter les deux garnitures de lit qui le composent. Les garnitures extérieures, au point de Hongrie, datent du début du $\mathrm{XVII}^{\mathrm{e}}$ siècle tandis que la garniture intérieure, courtepointe, dosseret, fond de lit et ciel de lit, est en satin de soie beige matelassé, brodé de fils de soie colorés et orné d'applications de velours et de passementeries. Dans le cadre du nouveau musée du Château de Foix, le lit est en cours d'étude et de restauration et sera présenté dans une period room évoquant une chambre d'apparat méridionale du $\mathrm{XVII}^{\mathrm{e}}$ siècle. Les conditions de présentation, et d'éclairage notamment, sont adaptées à la conservation préventive des textiles historiques.

Before becoming King of France, Henri IV was King of Navarre under the name of Henri III, Count of Foix. Although he never really resided in this county of Foix, he stayed there on several occasions between 1572 and 1578. Up to 1990, the Pailhès castle, situated to the north of the town of Foix, kept a remarkable seventeenth-century bed. The sovereign had a special relationship with Jacques de Villemur-Pailhès and appointed him governor of the county in 1566. So the creation of a bed chamber for the King in the castle is not altogether surprising, and bears witness to the prestige of this particular seigniory. Up to today, no document has been found to give us information as to the date of the construction of the bed or its installation. Held today by the Chateau de Foix, the bed underwent an important operation of restoration from 1994 to 1998 , dealing both with its bed frame structure and its textile components. The study of the bed shows that it was a composite object. The wooden frame was readapted at an unknown date and then again during its restoration, in order to adapt the two textile elements it comprises. The outer awning, with a herringbone pattern, dates from the beginning of the seventeenth century whilst the inner textiles-the quilt, backrest, the bed base and the canopy covering-are in padded silk satin embroidered with coloured silk thread and decorated with velvet applications and ornamental trimmings. A new museum is presently being prepared at the Chateau de Foix and the bed is being studied and restored again to be exhibited in a period room which will evoke a stately bed chamber in the south of France in the seventeenth century. The way the bed will be exhibited and lit will of course be properly adapted to the conservation requirements of the textiles.

\section{INDEX}

Mots-clés : lit, Ariège, Pailhès, Henri de Navarre, Henri IV, château de Foix, point de Hongrie, broderie, passementerie, rideau, pente haute, pente basse, ciel de lit, courtepointe, châlit, dais, restauration, textiles, éclairage, period room

Keywords : bed, Ariège, Pailhès, Henri de Navarre, Henri IV, Chateau de Foix, herringbone pattern, embroidery, ornamental trimmings, curtain, high slope, low slope, bed canopy, quilt, bedstead, dais, restoration, textiles, lighting, period room

\section{AUTEUR}

INGRID LEDUC

Conservateur départemental, Conseil départemental de l'Ariège ileduc@ariege.fr 